\title{
Computed Tomography of the Chest in COVID-19: A Pictorial Review of Indian Patients
}

\author{
Kumble Seetharama Madhusudhan ${ }^{1}$ \\ Niranjan Khandelwal ${ }^{2}$ \\ ${ }^{1}$ Department of Radiodiagnosis and Interventional Radiology, All \\ India Institute of Medical Sciences, New Delhi, India \\ 25C Network Private Limited, Bengaluru, Karnataka, India
}

\begin{abstract}
Address for correspondence Kumble S. Madhusudhan, MD, FRCR, Department of Radiodiagnosis and Interventional Radiology, All India Institute of Medical Sciences, Ansari Nagar, New Delhi 110029, India (e-mail: drmadhuks@gmail.com).
\end{abstract}

Ann Natl Acad Med Sci (India) 2021;57:82-92.

\begin{abstract}
Keywords

- severe acute respiratory syndrome

- coronavirus disease

- COVID-19

- consolidation

- ground glass opacity

The Coronavirus disease 2019, caused by severe acute respiratory syndrome corona virus 2 (SARS-CoV-2), has evolved into a pandemic and has affected more than 130 million people globally to date and continues to infect more. The disease primarily involves the respiratory system and manifests as fever, dry cough, dyspnea, and myalgia. Nearly half of the infected patients may be asymptomatic. The real-time reverse transcriptase polymerase chain reaction (RT-PCR) performed on the blood or respiratory samples is the diagnostic test with high accuracy. Although imaging with $C T$ is not routinely indicated in this disease, this modality may provide a quick answer and assist in making a diagnosis in certain situations. In addition, imaging with CT also aids in evaluating the progress of the disease and in prognostication. A thorough knowledge of the common findings on the $\mathrm{CT}$ scan helps a radiologist in suggesting a diagnosis when it is performed in unsuspected patients. In this review, we describe the common and uncommon chest findings of COVID-19 on the CT scan.
\end{abstract}

\section{Introduction}

The novel Coronavirus, causing severe acute respiratory syndrome (SARS)-like disease, is a recently discovered zoonotic virus spread from the bats, which act as natural hosts and reservoirs. ${ }^{1,2}$ This virus, now renamed as SARS Coronavirus-2 (SARS-CoV-2), belongs to the subgenus Sarbecovirus and family Coronaviridae and is a single-stranded RNA virus. ${ }^{2}$ The disease began in Wuhan, in China, in late December 2019 and was declared a pandemic by the World Health Organization (WHO), as it has spread to almost every country. To date, (April 04, 2021), the virus has infected over 130 million people worldwide and has resulted in over 2.8 million deaths. ${ }^{3}$

The virus predominantly affects the lungs and airways, and imaging is not routinely necessary for the diagnosis. However, many studies have shown that CT has a role to play

published online May 25, 2021
DOI https://doi.org/

$10.1055 / \mathrm{s}-0041-1728973$ ISSN 0379-038X in the diagnosis of COVID-19 in specific situations and hence the radiologist may be the first to suggest the diagnosis when it not clinically suspected.

\section{Clinical Features}

The SARS-CoV-2 virus primarily affects the respiratory system; particularly the lower respiratory tract. ${ }^{4}$ The most common symptom is fever, seen in up to $98 \%$ of the patients. Other common symptoms include dry cough, dyspnea, fatigue, and myalgia. ${ }^{4,5}$ Sputum production, headache, hemoptysis, and diarrhea are uncommon. The symptoms differ from the other diseases caused by Coronavirus family like SARS and Middle East respiratory syndrome (MERS), in that these infections more commonly affect the upper respiratory tract and have higher incidence of diarrhea. ${ }^{4}$ Although the disease affects all

C2021. National Academy of Medical Sciences (India).

This is an open access article published by Thieme under the terms of the Creative Commons Attribution-NonDerivative-NonCommercial-License, permitting copying and reproduction so long as the original work is given appropriate credit. Contents may not be used for commercial purposes, or adapted, remixed, transformed or built upon. (https://creativecommons.org/licenses/by-nc-nd/4.0/).

Thieme Medical and Scientific Publishers Pvt. Ltd. A-12, 2nd Floor, Sector 2, Noida-201301 UP, India 
ages and gender, it is more commonly seen in the middle age population and men. About $37 \%$ of the infected patients had associated comorbidities like diabetes mellitus, hypertension, and cardiovascular disease. ${ }^{5}$ About $30 \%$ of the patients may require admission to intensive care unit (ICU) to correct hypoxemia. Laboratory findings are nonspecific and show elevation of C-reactive protein (CRP), erythrocyte sedimentation rate (ESR), and lactate dehydrogenase (LDH). ${ }^{5}$

\section{Pathology}

The pathological changes in the lungs in patients with COVID-19 correlate with the stage of the disease and CT findings. ${ }^{6,7}$ The lung changes are found in almost all patients irrespective of whether the patient is symptomatic and are similar to that found in SARS and MERS. ${ }^{8,9}$ The primary pulmonary changes on histology are that of diffuse alveolar damage and include hyaline membrane deposition, alveolar epithelial cell injury, accumulation of exudates of fibrin, and diffuse hyperplasia of type II pneumocytes. ${ }^{10}$ The initial stage is mild exudative phase, which corresponds to ground-glass opacities (GGO) on CT scan. Histologically, there is alveolar edema, exudates of protein, focal areas of hyperplastic pneumocytes, and patchy infiltration of inflammatory cells. The subsequent stage is proliferative phase, which is represented by consolidation on CT scan and by fibroblast proliferation and deposition of extracellular matrix and fibrin within the alveoli on histology..$^{10}$ When there is secondary bacterial infection, the alveoli may show neutrophilic infiltration. The primary reason for the predominant pulmonary involvement in COVID-19 is the selective requirement of angiotensin-converting enzyme 2 (ACE-2) by the virus to infect cells and the high concentration of these receptors in the pneumocytes. ${ }^{11}$

\section{Diagnosis}

The disease is extremely contagious and has no specific treatment. These two factors make a timely diagnosis imperative to isolate and manage the infected person. ${ }^{12}$ The confirmatory test of COVID-19 is reverse transcriptase polymerase chain reaction (RT-PCR), which identifies the RNA in the respiratory or blood samples. The test has a sensitivity varying from $71 \%$ to $97 \%$ in different studies. ${ }^{13-15}$ The real time RT-PCR requires approximately 5 to 6 hours to produce the final result. In countries where the number of cases are high, the number of the kits for RT-PCR may not be readily available. ${ }^{16}$ This sometimes prompts the necessity of performing imaging, particularly CT scan, in clinically suspected patients of SARS-CoV-2 infection. Further, since nearly half of the patients are asymptomatic, incidental detection of findings on the lung bases may be possible when an abdominal scan is performed for an unrelated pathology. Hence, it is critical that the performing radiologist is clearly aware of the various chest CT appearances of COVID-19, so as to raise a suspicion of the disease and suggest proper subsequent steps in the management. The CT scan has a sensitivity for detection of the disease in the range of 86 to $98 \%$, but the specificity is low $(25 \%)$ as such findings may be seen in other infectious diseases. ${ }^{12,13}$

\section{Safety Measures with CT Scan}

COVID-19 is a highly contagious disease, and performing CT scan of patients afflicted with it exposes the staff and the scanner to contamination by the virus. All precautionary measures should be in place to avoid these risks. ${ }^{17}$ Prior to performing the CT scan, adequate staff training, properly laid standardized operative procedures like scheduling such patients during later part of the day and, whenever possible, dedicated hallways for these patients help in significantly mitigating the risk of transmission. Entry of personnel into the CT scanner room should be restricted and all, including patients, should wear a mask.

While performing the CT scan, the technologists should wear personal protective equipment (PPE). ${ }^{18}$ The recommendation is to have two technologists to perform a CT scan, one to remain in the scanning room to shift and position the patient and the other in the console room for planning and performing the scan. ${ }^{19}$

Once the CT scans are performed, the next step is decontamination of the CT scanner, the room, and room air. ${ }^{19}$ The protocol for decontamination is to mop the floor and the wipe the CT scanner, initially with water mixed with detergent, followed by sodium hypochlorite solution. Subsequently, the room is fumigated with formaldehyde for 30 minutes. The high-frequency contact surfaces should be wiped with a cloth soaked in alcohol-based disinfectants. The CT suite should be completely closed for 1 hour and properly ventilated.

\section{Technique of Computed Tomography}

The technique of performing a CT scan of the chest in a patient of COVID-19 is not different from the standard technique. The scan is performed with patient in supine position from thoracic inlet to costophrenic angles, with breath held in end-expiration. ${ }^{6}$ Intravenous (IV) contrast is usually not necessary. ${ }^{20}$ Both lung and mediastinal windows should be evaluated along with multiplanar reconstructions.

\section{Spectrum of Findings on Computed Tomography}

Various findings have been described on the CT scan of the chest in patients of COVID-19, which aid in the diagnosis. The spectrum of findings in the chest are broadly classified into four categories: pulmonary parenchymal, airway, pleural, and other organ changes. ${ }^{6,12,21,22}$ A schematic representation of the chest findings are shown in $\boldsymbol{- F i g .} \mathbf{1}$.

\section{Pulmonary Parenchymal Changes}

Pulmonary alveoli are the most common site of involvement in the chest. A variety of alterations in the alveoli and the interstitium have been described in patients with COVID-19 pneumonia. 


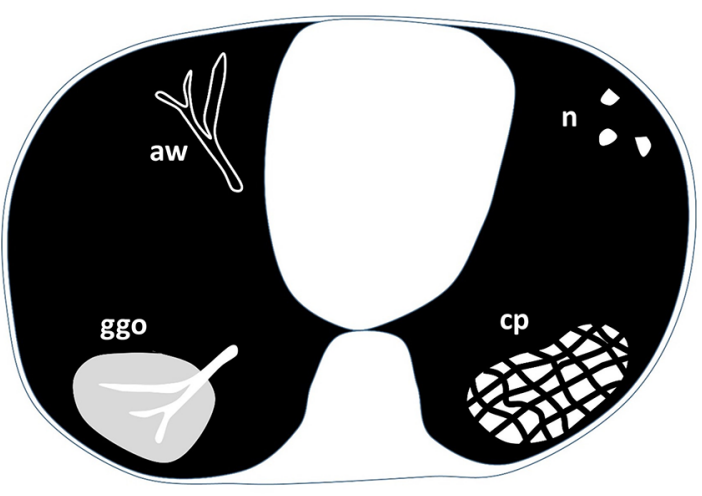

Early changes

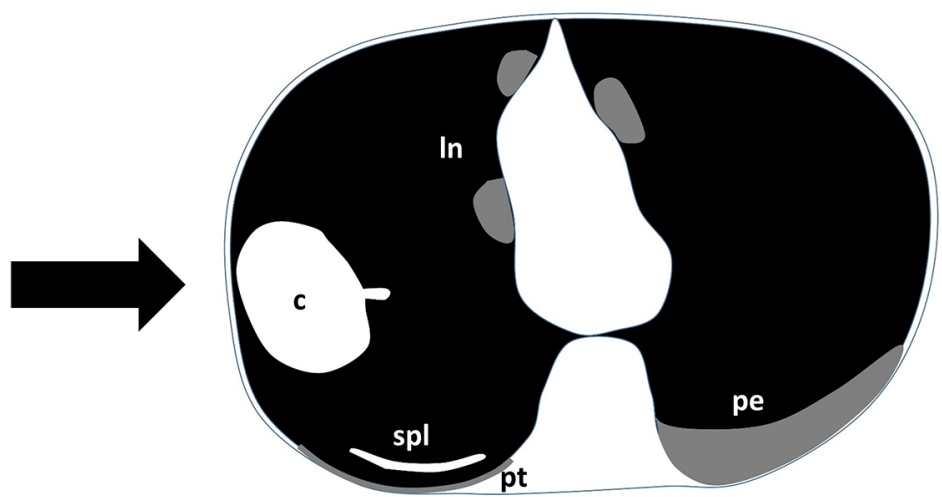

Late changes

Fig. 1 Schematic diagram of the changes in the lungs in COVID-19 pneumonia. aw-airway (bronchial wall thickening, bronchiectasis); c-consolidation (obscured vessels); cp-crazy paving (alveolar with interstitial exudates); ggo-ground-glass opacity (vasculature seen); In-mediastinal lymphadenopathy; n-nodules; pe-pleural effusion; pt-pleural thickening; spl-subpleural line.

\section{Ground-Glass Opacity}

Ground glass opacity (GGO) is the most common manifestation of COVID-19 pneumonia. The GGO occurs due to partial filling of the alveoli with the inflammatory exudates, fluid, and hyaline membrane. ${ }^{6,23}$ On CT scan, the GGO appear as an area of increased density in the lungs, without obscuration of vascular and bronchial markings (- Fig. 2).

It is considered as one of the earliest CT signs and is found in 98 to $100 \%$ of the patients. ${ }^{22,24,25}$ However, Pan et al suggested that the extent of the GGO and lung findings depends on the time of scan from the symptom onset, with GGO seen in small sizes and in subpleural regions in the early stage of the disease ( - Fig. 3). ${ }^{26}$ Subsequently, these progress to consolidation and interstitial thickening. ${ }^{27}$

\section{Consolidation}

Consolidation, the second most common finding in COVID-19 pneumonia, is seen as an area of increased density in the lungs, with obscuration of vascular and bronchial markings on the CT scan (-Fig. 4). Consolidation occurs when there is alveolar infiltration with fluid and inflammatory cells, as described in the previous section. ${ }^{10,21,23}$ This appearance is found in up to $63 \%$ of the patients and may be patchy, segmental, and multifocal. ${ }^{25,28}$ The consolidation may be found de novo or may develop in an area of previous GGO, indicating that this is seen more commonly as the disease progresses. ${ }^{6,24}$ Further, many of the patients during the course of the disease, particularly during 1 to 3 weeks, have a combination of GGO and consolidation. ${ }^{7}$ It may be associated with an air-bronchogram.

In a study by Song et al, the authors showed that consolidation is more common in the late stage of the disease and in patients aged more than 50 years. ${ }^{27}$ Hence, consolidation may be a warning of a severe disease course.

\section{Interstitial Changes}

Along with alveolar infiltration, there may be an associated infiltration of the intralobular and interlobular septae by lymphocytes, resulting in their thickening. ${ }^{6}$ This finding is seen in up to $49 \%$ of patients and constitutes the third most common finding. ${ }^{22}$ On the CT scan, the interstitial thickening is seen as reticular densities, which may have smooth or nodular outline ( - Fig. 5).6,21,22

A finding described along with interstitial tissue involvement is crazy paving pattern. This occurs due to coexisting GGO and interlobular septal thickening, giving the appearance of a
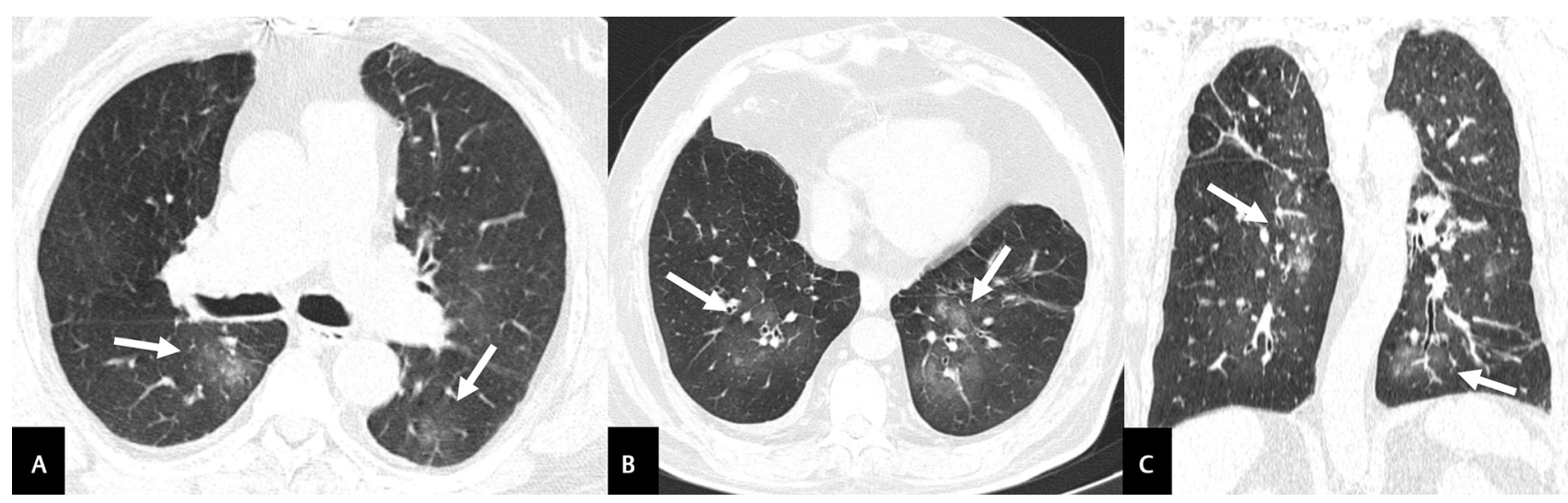

Fig. 2 Axial (A, B) and coronal (C) CT images in lung window of a 72-year-old male COVID-19 patient show multifocal ground-glass opacities (GGOs) in bilateral lungs, predominantly in the lower lobes (arrows). 
stoned pavement with random lines ( - Fig. 6). This finding is found in 5 to $36 \%$ of patients of COVID-19 pneumonia. ${ }^{29}$ When this finding is present with GGO and consolidation, it may suggest peak stage or progressive disease. ${ }^{26}$
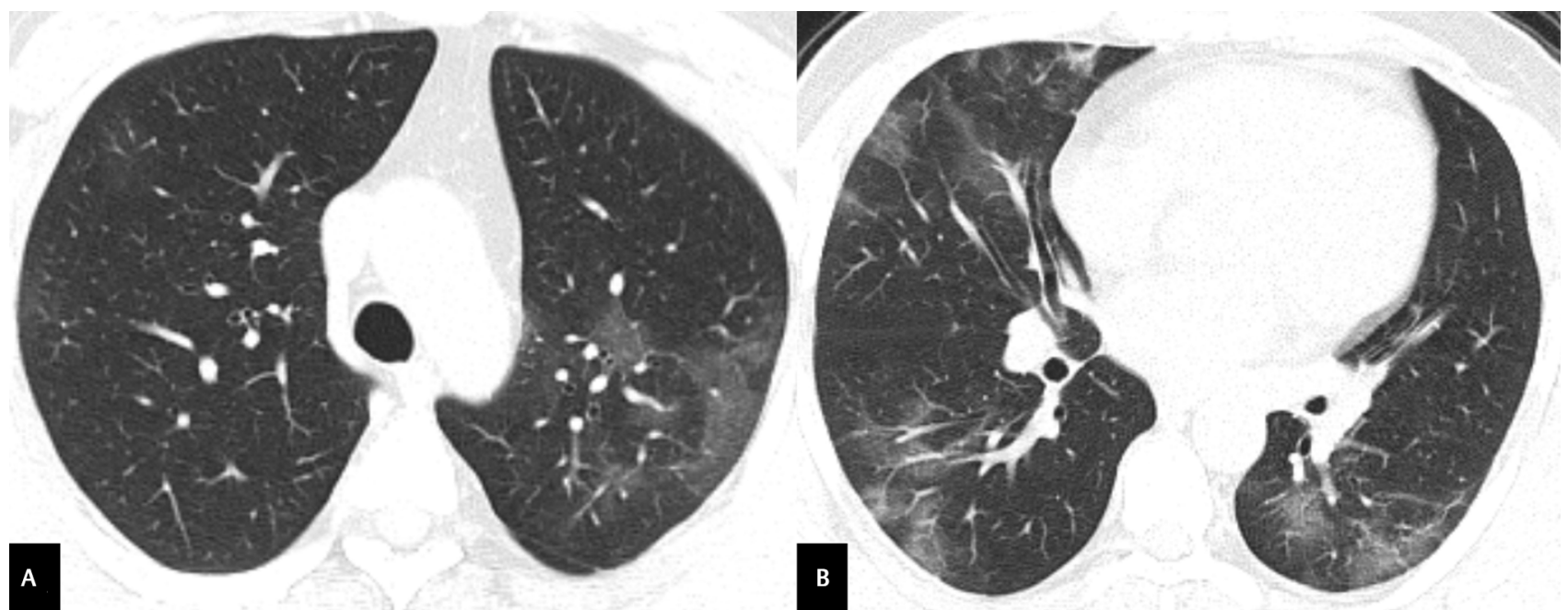

Fig. 3 Axial CT images in lung window (A, B) of a 40-year-old male with COVID-19 infection show multifocal ground-glass opacities (GGOs) in the peripheral and subpleural distribution.

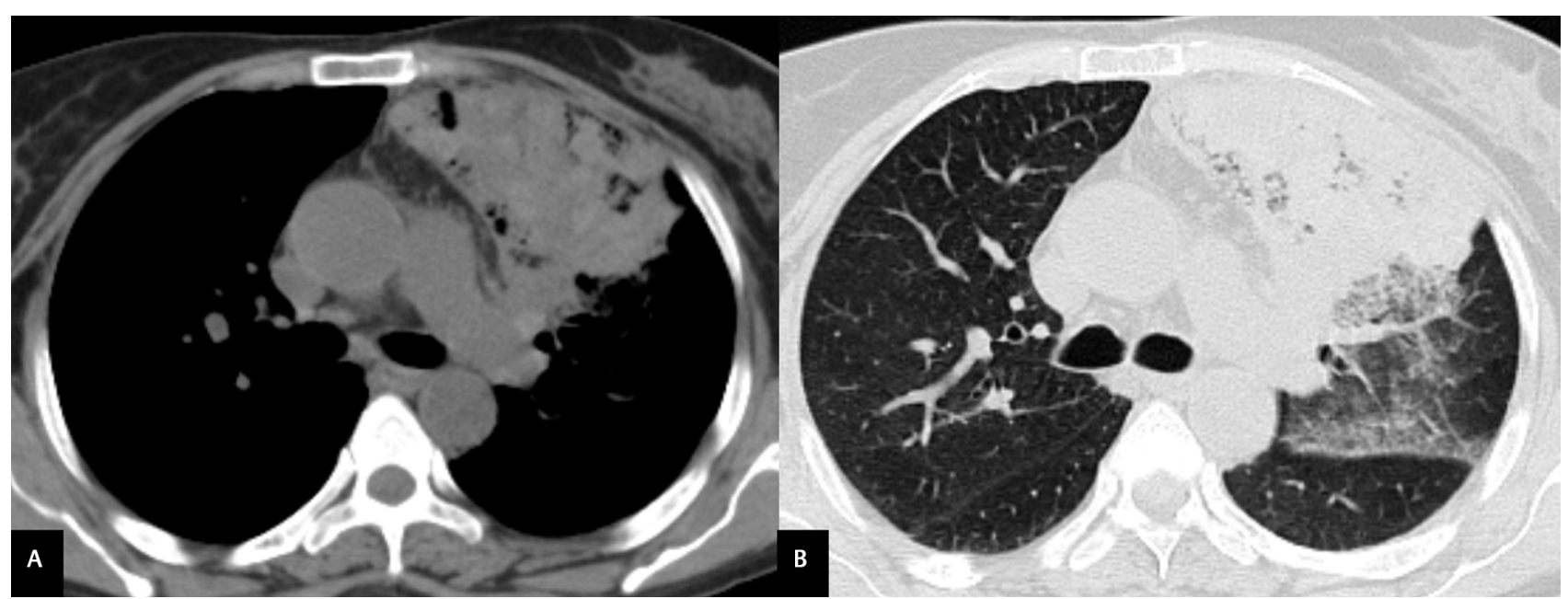

Fig. 4 Axial CT images, mediastinal (A) and lung (B) windows of a 64-year-old female with COVID-19 pneumonia show an area of segmental consolidation in the left upper lobe. Adjacent ground-glass opacities (GGOs) and interlobular septal thickening are also seen.

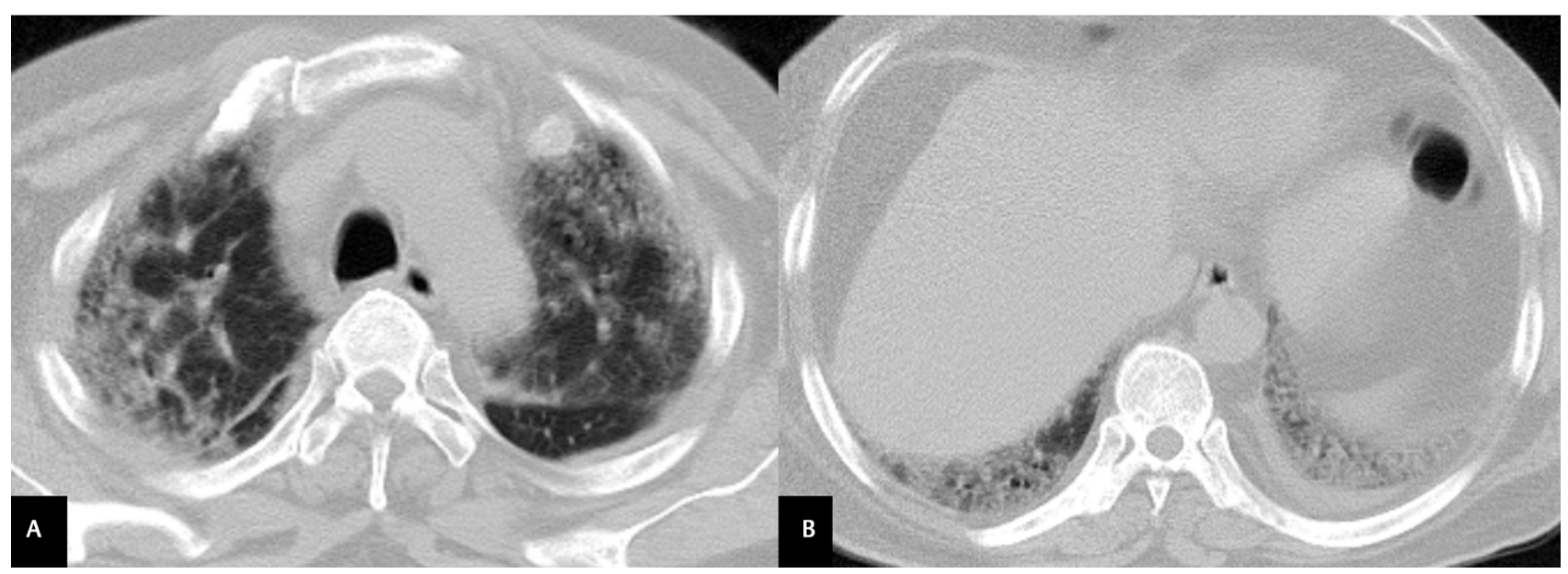

Fig. 5 Axial CT images in lung window (A, B) of a 55-year-old male with COVID-19 infection show areas of intralobular and interlobular septal thickening in the bilateral subpleural regions. 

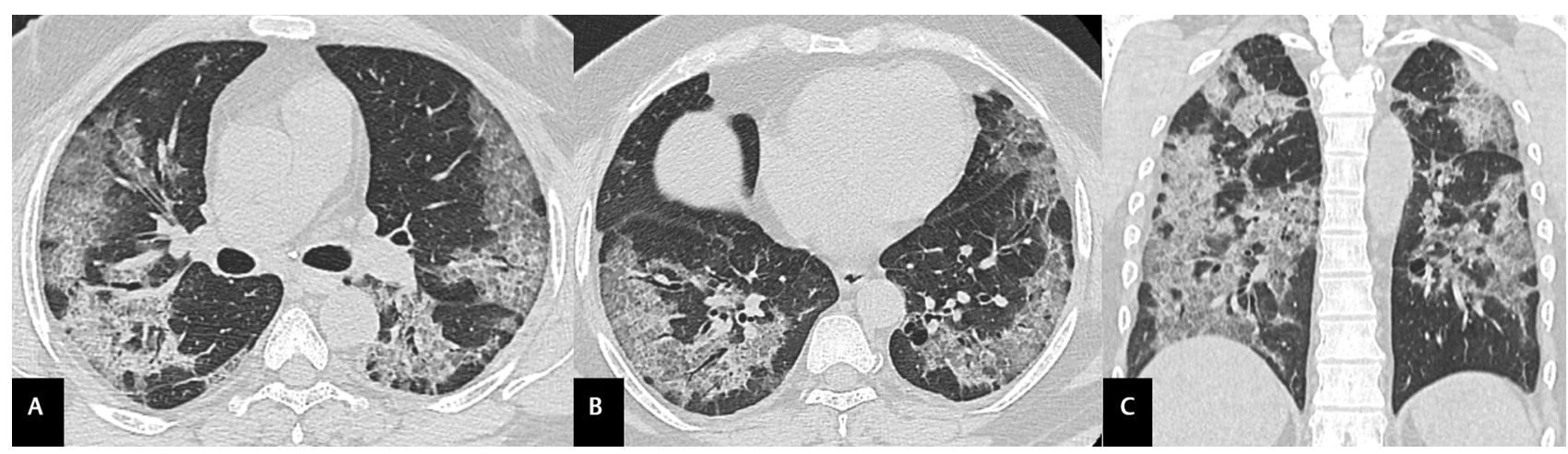

Fig. 6 Axial (A, B) and coronal (C) CT images of a 30-year-old male with COVID-19 infection show combination of ground-glass opacities (GGOs) and interstitial thickening in bilateral lungs, giving the appearance of crazy paving.
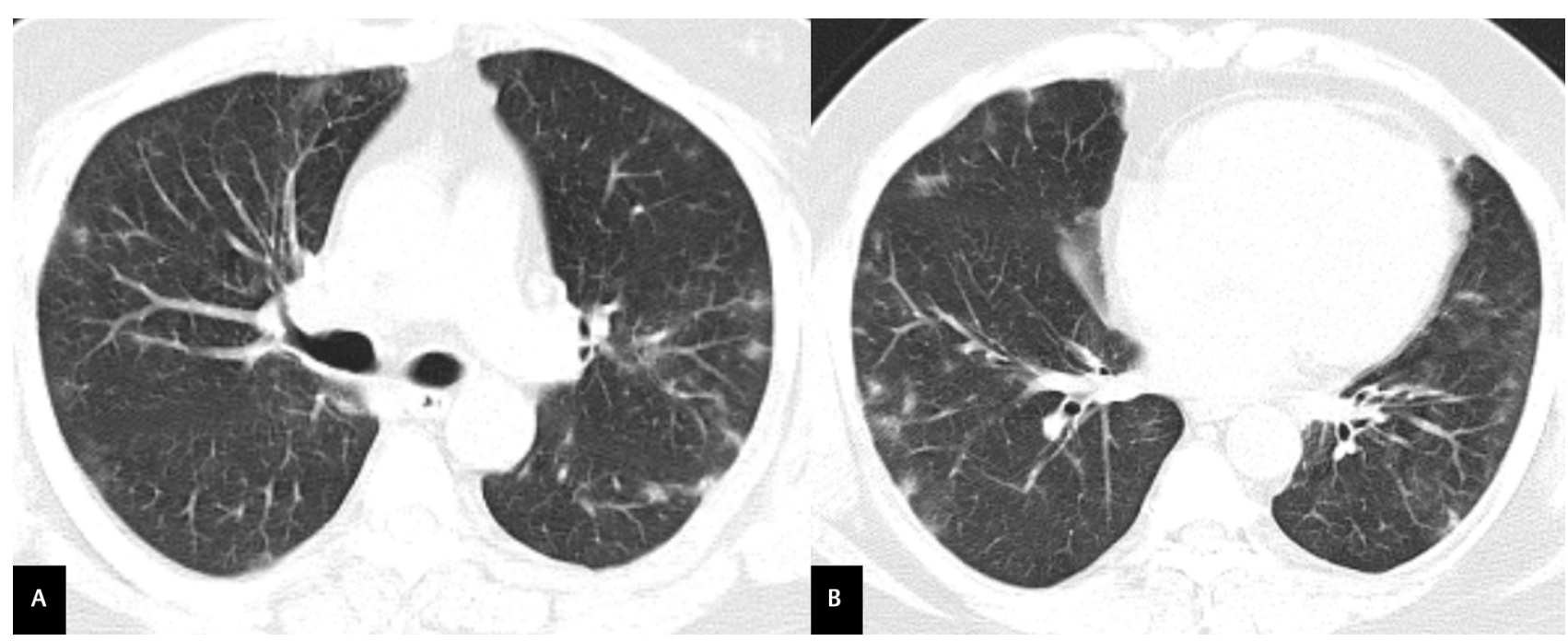

Fig. 7 Axial CT images in lung windows (A, B) of a 55-year-old male with COVID-19 infection show ill-defined centrilobular nodules in bilateral lungs, predominantly distributed in the subpleural regions.

\section{Nodules}

About $23 \%$ of patients with COVID-19 may show centrilobular nodules (size $<3 \mathrm{~cm}$ ) on CT scan. ${ }^{22}$ The nodules may be solitary or, more commonly, multiple and frequently have ill-defined margins ( - Fig. 7 ). ${ }^{21}$ As the disease progresses, the nodules may increase in size and number. ${ }^{30}$ There may be an associated ground glass halo around some of the nodules.

\section{Vascular Enlargement}

Enlargement of the pulmonary vasculature is found in approximately $70 \%$ of the patients of COVID- $19 .{ }^{22}$ On CT scan, they are seen as smoothly dilated pulmonary vessels within or around an area of GGO or consolidation ( - Fig. 8). This finding develops due to the vasodilatation, injury and edema of the vessel wall from acute inflammation..$^{21,22}$

\section{Halo and Reverse Halo Sign}

Halo sign is defined as a nodule with surrounding GGO (-Fig. 9). ${ }^{23}$ This finding is nonspecific and is uncommonly described in COVID-19 pneumonia. ${ }^{31,32}$ Although in other diseases, the halo of GGO is due to hemorrhage, its pathogenesis in COVID-19 is currently unclear.
A reverse halo or atoll sign has also been described in patients of COVID-19. This sign is defined as a halo of consolidation around a low-density core or GGO ( - Fig. 9). ${ }^{33}$ They occur when a ring of consolidation develops around a GGO core or when there is healing in the center. ${ }^{25}$

Both these signs are uncommonly described in COVID-19 pneumonia. A study by Li et al found halo sign and reverse halo sign in $17.6 \%$ and $3.8 \%$ of patients, respectively. ${ }^{31}$

\section{Fibrosis and Architectural Distortion}

In the late stage of the disease, fibrosis and architectural distortion may be seen. This occurs as a result of the healing process of the GGO, consolidation, and interstitial disease. ${ }^{30}$ On CT scan, fibrosis is seen as irregular sharply marginated densities, in the alveolar space or interstitium, or both, leading to distortion of the parenchymal structure, lobar or pulmonary volume loss, and mediastinal shift ( - Fig. 10). ${ }^{20}$ The incidence of fibrosis on CT scan varies from 21 to $53 \%{ }^{20,22}$ Although fibrosis indicates healing, its prognosis is still not clear. The reversibility of the fibrosis is not currently known. 

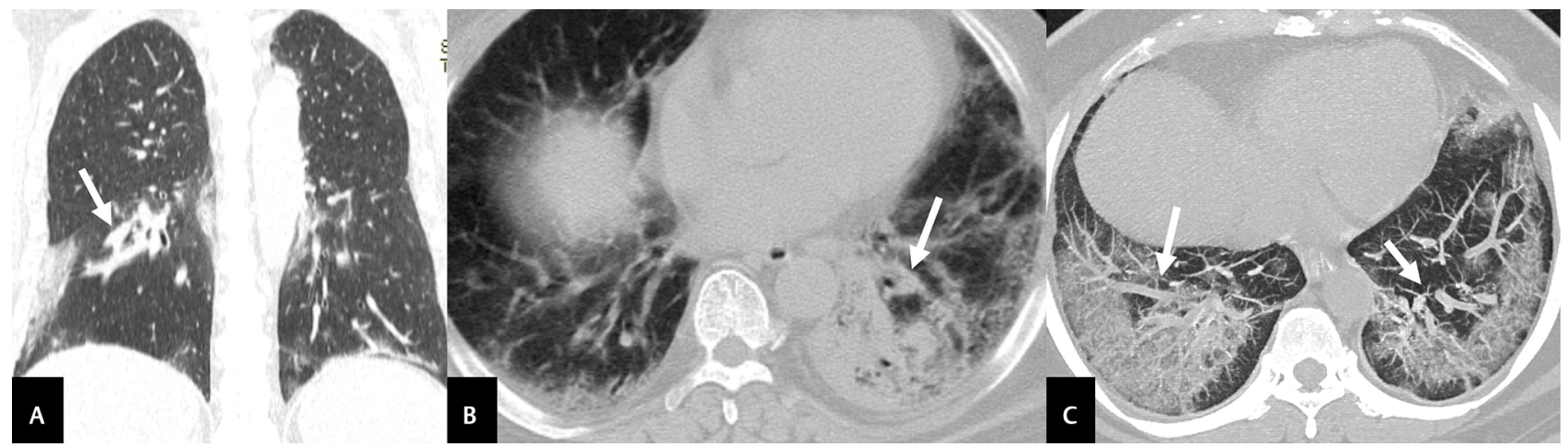

Fig. 8 CT images in lung windows of three different patients of COVID-19 pneumonia showing vascular enlargement in association with ground-glass opacities (GGOs) (arrow in A), consolidation (arrow in $\mathbf{B}$ ) and crazy-paving (arrows in $\mathbf{C}$ ).

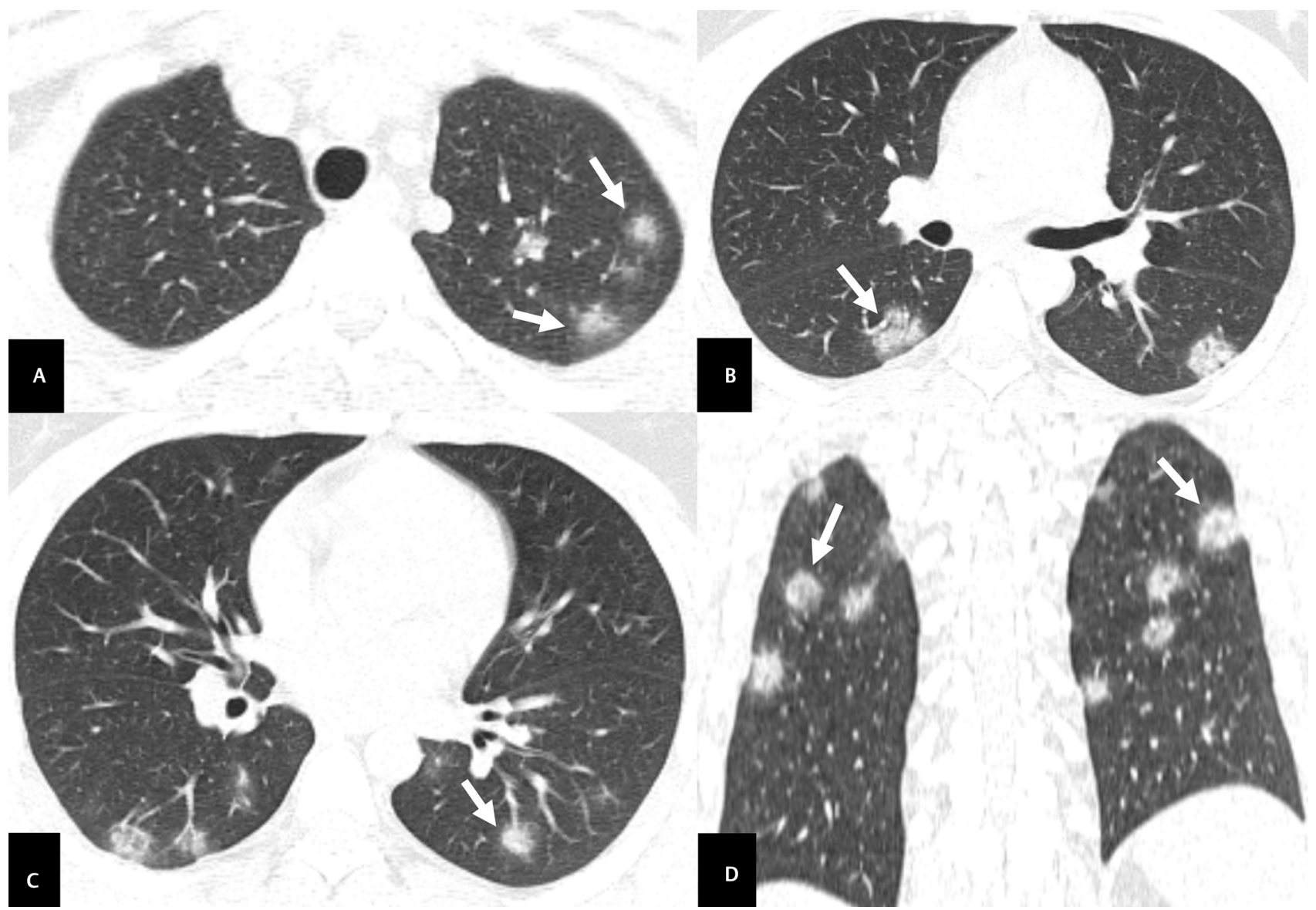

Fig. 9 Axial (A-C) and coronal (D) CT images in lung window of a 25-year-old male with COVID-19 pneumonia show multiple nodules in bilateral lungs, with halo sign (arrows in $\mathbf{A}-\mathbf{C}$ ) and reverse halo sign (arrows in $\mathbf{D}$ ).

\section{Distribution}

Many studies describing the distribution of the pulmonary findings have found that the most common site of involvement is the lower lung lobe, seen in 55\% of the cases ( - Fig. 11).22 Diffuse involvement of upper and lower lobes is the second most common appearance, and in $6 \%$, upper lobes are predominantly involved. Peripheral and subpleural pulmonary areas are more commonly involved (75\%) compared to the central regions ( - Fig. 11).22,24 In $80 \%$, the disease is bilateral, and in $55 \%$, it is multifocal.

\section{Airway Changes}

The airway changes which are described in COVID-19 include bronchial wall thickening and bronchiectasis. ${ }^{21}$

\section{Bronchial Wall Thickening}

Bronchial wall thickening, which occurs due to inflammation of the bronchial wall, is found in less than a third of patients of COVID-19..22,28 On CT scan, it is seen as a smoothly thickened walls of the bronchi $(\boldsymbol{- F i g}$. 12). One study by Li et al 

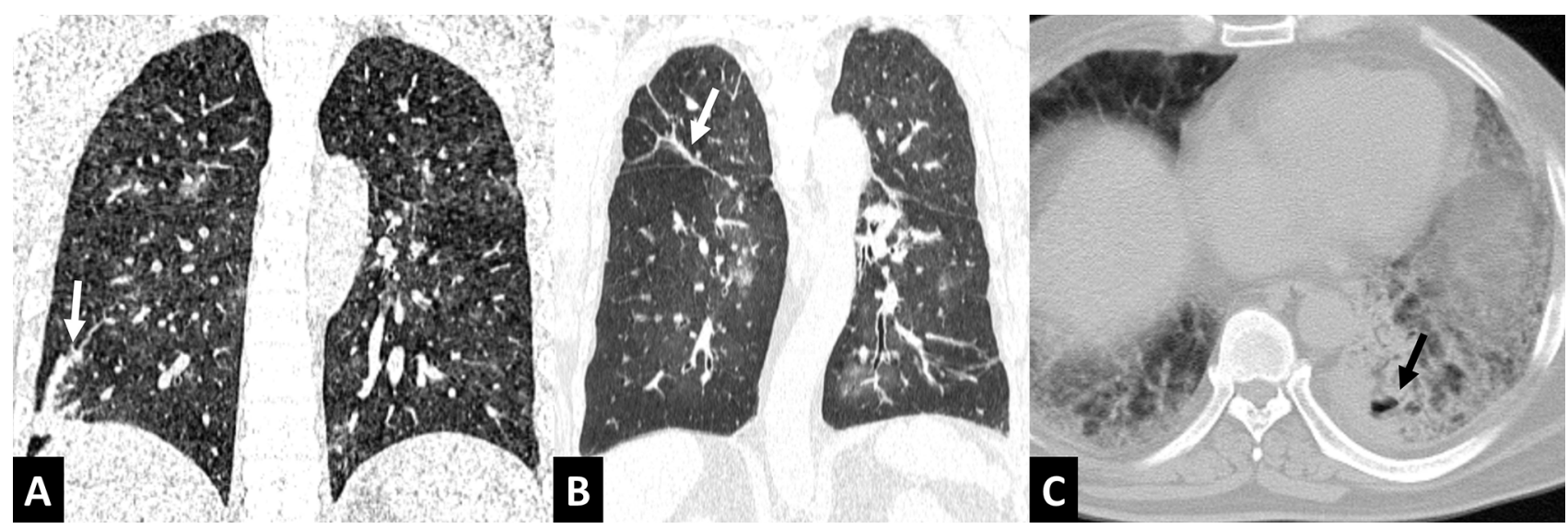

Fig. 10 Coronal (A, B) and axial (C) CT images in lung window of three different patients of COVID-19 pneumonia in recovery phase show fibrotic bands (arrows in $\mathbf{A}$ and $\mathbf{B}$ ) and cavitation (arrow in $\mathbf{C}$ ).

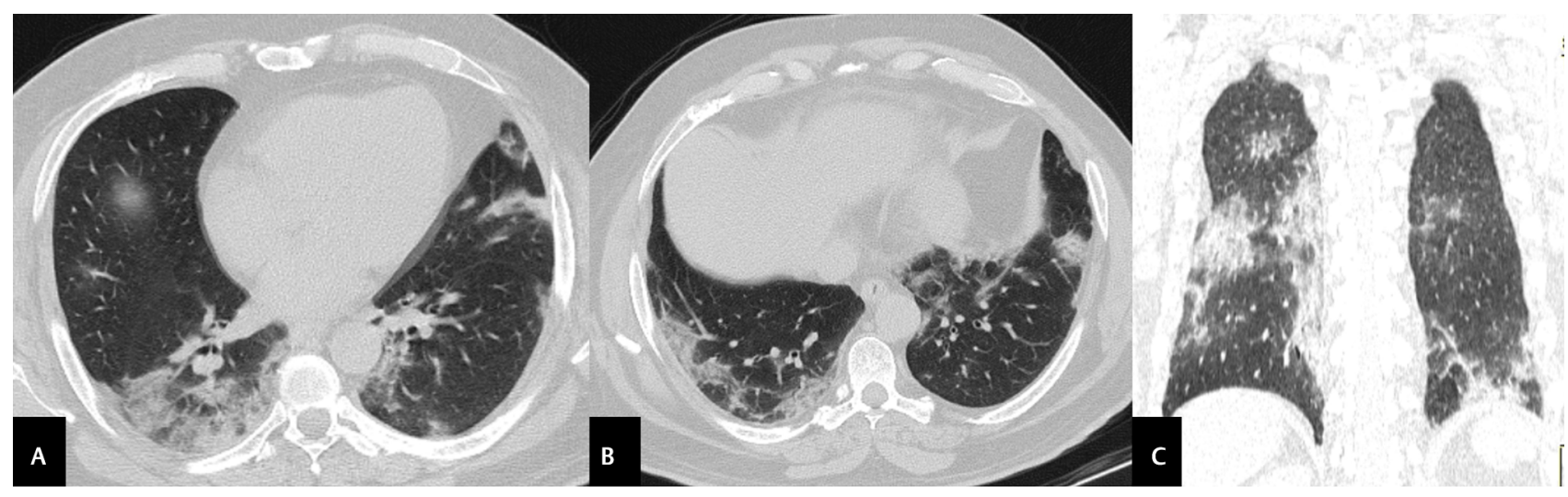

Fig. 11 Axial (A, B) and coronal (C) CT images in lung window of a 60-year-old male with COVID-19 pneumonia show the distribution of the pulmonary involvement, which is multifocal, peripheral and subpleural with lower lobar predominance.

has shown that this finding was more common in severe and critically ill patients, which may help in predicting disease severity and prognosis. ${ }^{25}$

\section{Bronchiectasis}

Bronchiectasis or bronchiolectesis, defined as abnormal irreversible dilatation of the airways, occurs either due to inflammatory weakening of the bronchial wall or due to traction of the bronchi by surrounding fibrosis (tractional bronchiectasis) (-Fig. 12). ${ }^{21}$ Bronchiectasis is found during the second and third week of the disease and may indicate the beginning of recovery process. ${ }^{6}$

Air bubble sign is a rare finding described in COVID-19 pneumonia. $^{21}$ On CT scan, it is seen as a small air-containing space or cyst, which could be due to bronchiolectasis or resorption of an area of consolidation ( - Fig. 12).

\section{Pleural Changes}

\section{Pleural Effusion}

Pleural effusion is an infrequent finding in COVID-19 and is seen in approximately 5 to $14 \%$ of the patients $\left(-\right.$ Fig. 13 ) ${ }^{6,22}$ This finding is seen late in the course of the disease and may be associated with poor prognosis. ${ }^{20}$

\section{Pleural Thickening}

Pleural thickening is more common than pleural effusion and is seen in $32 \%$ of patients ( Fig. 13) ${ }^{6,7}$ This finding is usually associated with pulmonary signs like GGO, consolidation, or interstitial thickening.

\section{Subpleural Lines}

Subpleural lines or bands, another relatively common finding, are seen in less than a third of patients. On CT scan, these lines are seen within $1 \mathrm{~cm}$ of the pleural surface and appear as 1 - to 3-mm thick curvilinear densities ( - Fig. 13). ${ }^{21}$ This finding may be related to either pulmonary edema or fibrosis. ${ }^{25}$

\section{Other Changes}

\section{Lymphadenopathy}

Mediastinal lymphadenopathy is an infrequent finding in COVID-19 and has been reported in 4 to $8 \%$ of patients (-Fig. 14). ${ }^{7,28}$ This finding is associated with severe and critical pneumonia and is considered to be a poor prognostic factor. ${ }^{25}$ However, secondary bacterial infection should be considered when this finding is found in association with lung nodules and pleural effusion. ${ }^{12,13}$ 


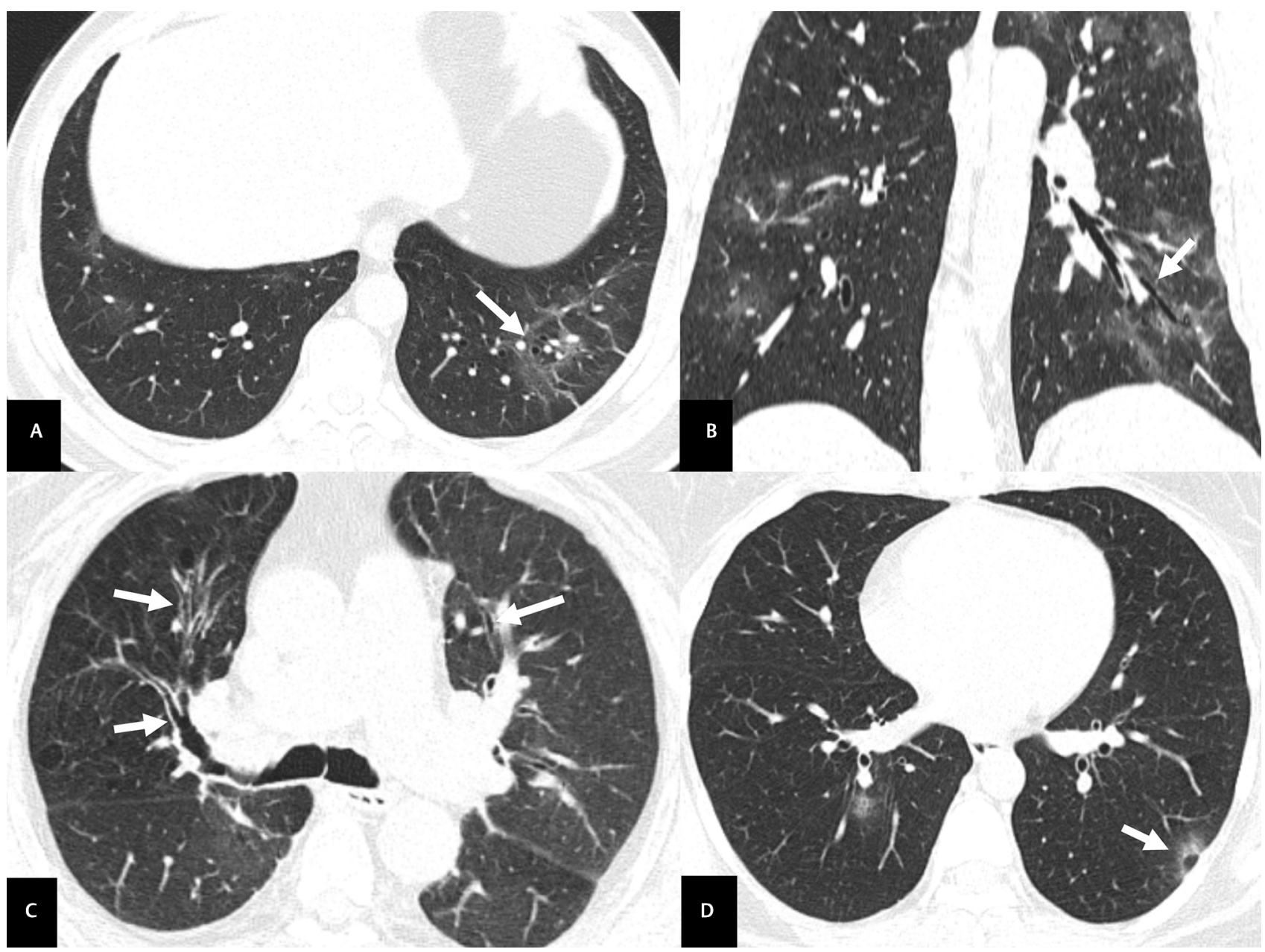

Fig. 12 A, B-Axial (A) and coronal (B) CT images in lung window of a 40-year-old male with COVID-19 infection show bronchiectasis (arrow) with surrounding ground-glass opacities (GGOs). C-Axial CT image in lung window of a 72-year-old male with COVID-19 shows bronchial wall thickening bilaterally (arrows). D-Axial CT image in lung window of a 35-year-old female with COVID-19 pneumonia shows the characteristic cyst sign (arrow).

\section{Pericardial Effusion}

Pericardial effusion is found in approximately $5 \%$ of the patients of COVID-19 and is frequently seen in association with severe and critical disease. ${ }^{24}$ Thus, this finding also carries a poorer prognosis. ${ }^{25}$

\section{Pulmonary Embolism}

Some reports have suggested the development of pulmonary embolism (PE) in patients of COVID-19 presenting with respiratory failure. ${ }^{34,35}$ The current reports have indicated that pulmonary embolism is seen in 22 to $30 \%$ patients afflicted with COVID-19. ${ }^{35}$ CT pulmonary angiography should be performed whenever PE is clinically suspected.

\section{Recovery Phase of COVID-19}

The recovery process typically starts after 14 days of the disease in about three-fourths of the patients. ${ }^{24}$ During the recovery phase of the disease, the CT scan shows improvement in the previously documented findings in the form of reduction in the extent of pulmonary or lobar involvement, resorption of GGO and consolidation and disappearance of nodules and crazy paving feature. ${ }^{26}$ Subsequently, cavities may develop along with bronchiectasis and other fibrotic changes. The long-term consequences of fibrotic changes are currently not known.

\section{Differential Diagnosis}

As mentioned in a previous section, although CT scan has high sensitivity of up to $98 \%$, its specificity is only $25 \%{ }^{12}$ This is because the findings seen on CT scan are found in many other diseases caused by adenovirus, influenza and parainfluenza viruses, rhinovirus, and respiratory syncytial virus. ${ }^{31,36}$ Although difficult, based on the predominantCT findings, it may be possible to differentiate COVID-19 pneumonia from other viral pneumonias. These findings include asymmetrically distributed centrilobular nodules and consolidation in respiratory syncytial virus; bilateral multifocal, lobar or segmental GGO and consolidation in adenovirus; 


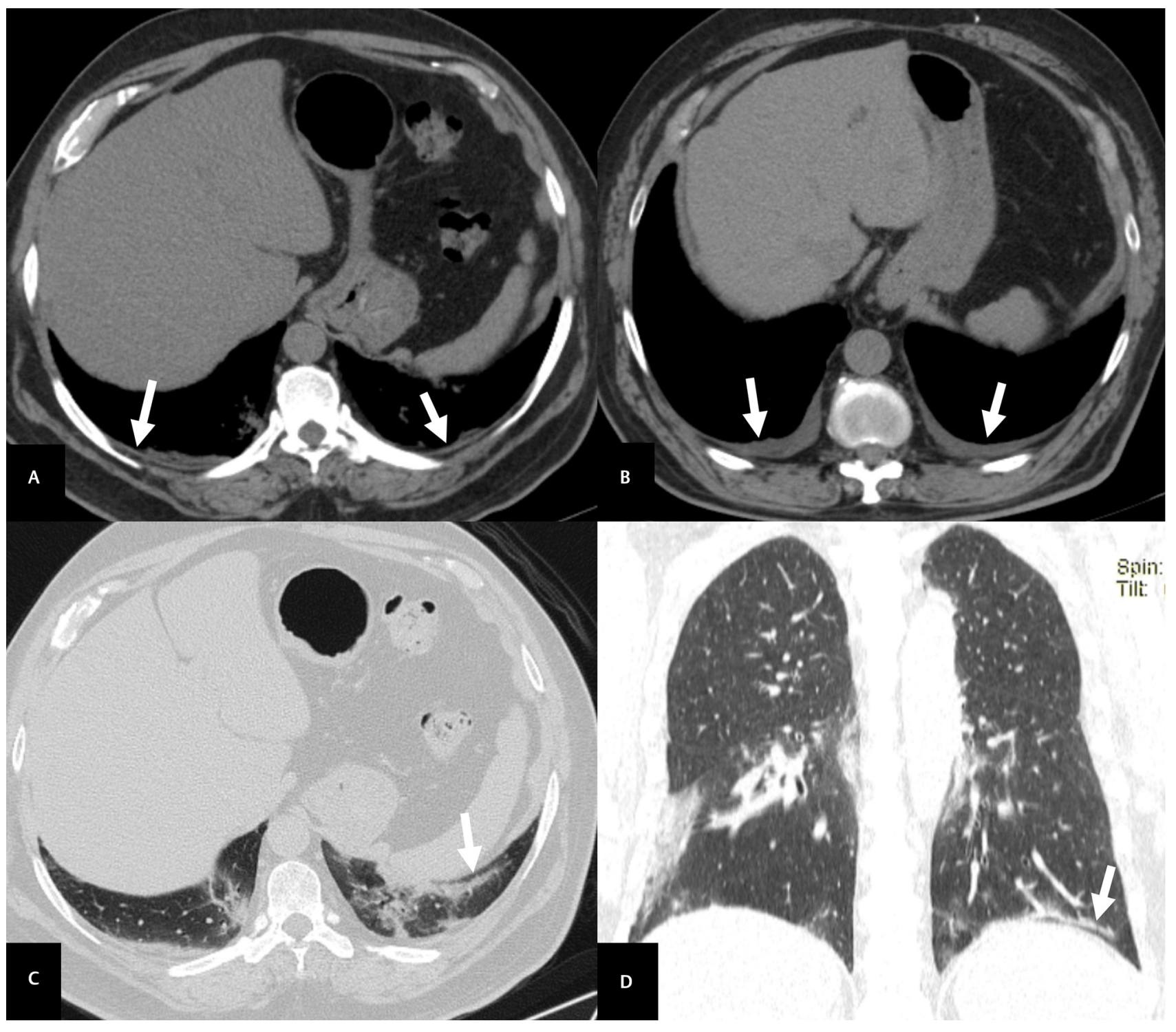

Fig. 13 Axial (A, B-mediastinal window; C-lung window) and coronal (D-lung window) CT images of three different patients with COVID-19 pneumonia show pleural involvement in the form of bilateral pleural thickening (arrows in A), bilateral pleural effusion (arrows in $\mathbf{B}$ ), and subpleural band (arrow in $\mathbf{C}$ and $\mathbf{D}$ ).

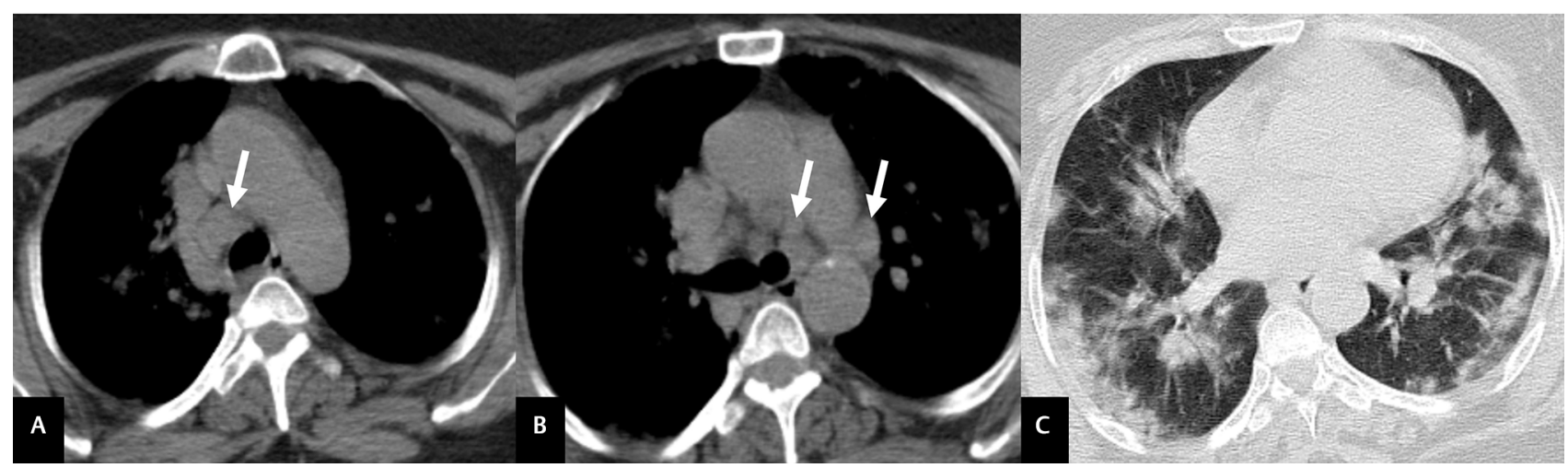

Fig. 14 Axial CT images in mediastinal (A, B) and lung (C) windows show enlarged mediastinal lymph nodes (arrows in $\mathbf{A}$ and $\mathbf{B}$ ), with multifocal ground-glass opacities (GGOs) in bilateral lungs. 
centrilobular nodules and bronchial wall thickening in parainfluenza virus; and bilateral, lower lobar GGO and consolidation in influenza virus pneumonias. ${ }^{37}$

In addition to the above, two other differentials include SARS and MERS, as both these diseases are caused by other Coronaviruses and have similar pathological changes. ${ }^{21}$ However, some imaging findings are found to be more common in COVID-19 pneumonia compared to MERS and SARS. These include predominant multifocal involvement, nodules with halo sign and reverse halo sign. ${ }^{31,38}$ In addition, some findings like lymphadenopathy, pleural effusion, nodules, and cavitations are uncommon in SARS and MERS. ${ }^{38}$

\section{Conclusion}

In conclusion, COVID-19 is a highly contagious viral infection, principally affecting the respiratory system. Although, the real-time RT-PCR is highly accurate in making the diagnosis, CT scan helps in assessing the disease severity and in prognostication. Adequate precautions should be taken while performing CT scan to avoid cross-contamination. Knowledge of the spectrum of findings on the CT scan, as described in this review, is helpful in raising suspicion of the disease in unsuspected cases and differentiating COVID-19 pneumonia from other viral diseases to some extent.

\section{Conflict of Interest}

None declared.

\section{Acknowledgment}

The authors thank 5C Network Private Limited for their assistance in providing quality images.

\section{References}

1 Bonilla-Aldana DK, Dhama K, Rodriguez-Morales AJ. Revisiting the one health approach in the context of COVID-19: a look into the ecology of this emerging disease. Adv Anim Vet Sci 2020;8:234-237

2 Zhu N, Zhang D, Wang W, et al. China Novel Coronavirus Investigating and Research Team. A Novel coronavirus from patients with pneumonia in China, 2019. N Engl J Med 2020; 382(8):727-733

3 WHO coronavirus disease (COVID-19) situation dashboard. Available at: https://covid19.who.int/. Accessed April 05, 2021

4 Huang C, Wang Y, Li X, et al. Clinical features of patients infected with 2019 novel coronavirus in Wuhan, China. Lancet 2020;395(10223):497-506

5 Rodriguez-Morales AJ, Cardona-Ospina JA, Gutiérrez-Ocampo E, et al; Latin American Network of Coronavirus Disease 2019-COVID-19 Research (LANCOVID-19). Electronic address: https://www.lancovid.org. Clinical, laboratory and imaging features of COVID-19: A systematic review and meta-analysis. Travel Med Infect Dis 2020;34:101623

$6 \mathrm{Xu} \mathrm{Z}$, Shi L, Wang Y, et al. Pathological findings of COVID-19 associated with acute respiratory distress syndrome. Lancet Respir Med 2020;8(4):420-422

7 Shi H, Han X, Jiang N, et al. Radiological findings from 81 patients with COVID-19 pneumonia in Wuhan, China: a descriptive study. Lancet Infect Dis 2020;20(4):425-434
8 Ding Y, Wang $\mathrm{H}$, Shen $\mathrm{H}$, et al. The clinical pathology of severe acute respiratory syndrome (SARS): a report from China. J Pathol 2003;200(3):282-289

9 Ng DL, Al Hosani F, Keating MK, et al. Clinicopathologic, immunohistochemical, and ultrastructural findings of a fatal case of Middle East respiratory syndrome coronavirus infection in the United Arab Emirates, April 2014. Am J Pathol 2016;186(3):652-658

10 Tian S, Xiong Y, Liu H, et al. Pathological study of the 2019 novel coronavirus disease (COVID-19) through postmortem core biopsies. Mod Pathol 2020;33(6):1007-1014

$11 \mathrm{Xu} \mathrm{X}$, Chen P, Wang J, et al. Evolution of the novel coronavirus from the ongoing Wuhan outbreak and modeling of its spike protein for risk of human transmission. Sci China Life Sci 2020;63(3):457-460

12 Ai T, Yang Z, Hou H, et al. Correlation of Chest CT and RT-PCR Testing for Coronavirus Disease 2019 (COVID-19) in China: A Report of 1014 Cases. Radiology 2020;296(2):E32-E40

13 Fang Y, Zhang H, Xie J, et al. Sensitivity of Chest CT for COVID19: Comparison to RT-PCR. Radiology 2020;296(2):E115-E117

14 Long C, Xu H, Shen Q, et al. Diagnosis of the Coronavirus disease (COVID-19): rRT-PCR or CT? Eur J Radiol 2020;126:108961

15 Mossa-Basha M, Meltzer CC, Kim DC, Tuite MJ, Kolli KP, Tan BS. Radiology Department Preparedness for COVID19: Radiology Scientific Expert Review Panel. Radiology 2020;296(2):E106-E112

16 Udugama B, Kadhiresan P, Kozlowski HN, et al. Diagnosing COVID-19: The Disease and Tools for Detection. ACS Nano 2020;14(4):3822-3835

17 Kooraki S, Hosseiny M, Myers L, Gholamrezanezhad A. Coronavirus (COVID-19) outbreak: what the Department of Radiology should know. J Am Coll Radiol 2020;17(4):447-451

18 Mossa-Basha M, Medverd J, Linnau KF, et al. Policies and Guidelines for COVID-19 Preparedness: Experiences from the University of Washington. Radiology 2020;296(2):E26-E31

19 Nakajima K, Kato H, Yamashiro T, et al. COVID-19 pneumonia: infection control protocol inside computed tomography suites. Jpn J Radiol 2020;38(5):391-393

20 Li K, Fang Y, Li W, et al. CT image visual quantitative evaluation and clinical classification of coronavirus disease (COVID-19) Eur Radiol 2020;30(8):4407-4416

21 Ye Z, Zhang Y, Wang Y, Huang Z, Song B. Chest CT manifestations of new coronavirus disease 2019 (COVID-19): a pictorial review. Eur Radiol 2020;30(8):4381-4389

22 Zhao W, Zhong Z, Xie X, Yu Q, Liu J. Relation between chest CT findings and clinical conditions of Coronavirus disease (COVID-19) pneumonia: a multicenter study. Am J Roentgenol 2020;214(5):1072-1077

23 Hansell DM, Bankier AA, MacMahon H, McLoud TC, Müller NL, Remy J. Fleischner Society: glossary of terms for thoracic imaging. Radiology 2008;246(3):697-722

24 Salehi S, Abedi A, Balakrishnan S, Gholamrezanezhad A. Coronavirus disease 2019 (COVID-19): a systematic review of imaging findings in 919 patients. Am J Roentgenol 2020; 215(1):87-93

$25 \mathrm{Li} \mathrm{K}, \mathrm{Wu}$ J, Wu F, et al. The clinical and chest CT features associated with severe and critical COVID-19 pneumonia. Invest Radiol 2020;55(6):327-331

26 Pan F, Ye T, Sun P, et al. Time course of lung changes on chest CT during recovery from 2019 novel coronavirus (COVID19) pneumonia. Radiology 2020;Doi: https://doi.org/10.1148/ radiol.2020200370

27 Song F, Shi N, Shan F, et al. Emerging coronavirus 2019- nCoV pneumonia. Radiology 2020;Doi: 10.1148/radiol.2020200274

$28 \mathrm{Wu} \mathrm{J}, \mathrm{Wu} \mathrm{X}$, Zeng W, et al. Chest CT findings in patients with coronavirus disease 2019 and its relationship with clinical features. Invest Radiol 2020;55(5):257-261 
92 Chest CT in COVID-19 Madhusudhan et al.

29 Bernheim A, Mei X, Huang M, et al. Chest CT findings in coronavirus disease-19 (COVID-19): relationship to duration of infection. Radiology 2020;295(3):200463

30 Pan Y, Guan H, Zhou S, et al. Initial CT findings and temporal changes in patients with the novel coronavirus pneumonia (2019-nCoV): a study of 63 patients in Wuhan, China. Eur Radiol 2020;30(6):3306-3309

31 Li Y, Xia L. Coronavirus disease 2019 (COVID-19): role of chest CT in diagnosis and management. Am J Roentgenol 2020;214(6):1280-1286

32 Li X, Zeng X, Liu B, Yu Y. COVID-19 infection presenting with CT halo sign. Radiol Cardiothorac Imaging 2020;2(1):e200026

33 Huang P, Liu T, Huang L, et al. Use of chest CT in combination with negative RT-PCR assay for the 2019 novel coronavirus but high clinical suspicion. Radiology 2020;295(1):22-23

34 Tang N, Li D, Wang X, Sun Z. Abnormal coagulation parameters are associated with poor prognosis in patients with novel coronavirus pneumonia. J Thromb Haemost 2020;18(4):844-847
35 Oudkerk M, Büller HR, Kuijpers D, et al. Diagnosis, prevention, and treatment of thromboembolic complications in COVID19: report of the national institute for public health of the Netherlands. Radiology 2020;297(1):E216-E222

36 Bai HX, Hsieh B, Xiong Z, et al. Performance of radiologists in differentiating COVID-19 from viral pneumonia on chest CT. Radiology 2020;296(2):E46-E54

37 Carotti M, Salaffi F, Sarzi-Puttini P, et al. Chest CT features of coronavirus disease 2019 (COVID-19) pneumonia: key points for radiologists. Radiol Med (Torino) 2020;125(7):636-646

38 Hosseiny M, Kooraki S, Gholamrezanezhad A, Reddy S, Myers L. Radiology perspective of coronavirus disease 2019 (COVID-19): lessons from severe acute respiratory syndrome and middle east respiratory syndrome. Am J Roentgenol 2020;214(5):1078-1082 